\title{
THEORETICAL ASPECTS OF THE FIREBALL SCENARIO
}

\author{
A. Bret ${ }^{1}$, A. Stockem ${ }^{2}$, E. Pérez-Álvaro ${ }^{1}$, F. Fiuza ${ }^{2}$, C. Ruyer ${ }^{3}$, \\ L. Gremillet ${ }^{5}$, R. Narayan ${ }^{4}$ and L.O. Silva ${ }^{2}$
}

\begin{abstract}
Collisionless shocks are a key ingredient of the Fireball scenario. Yet, their formation from the encounter of two collisionless plasma shells is not understood from first principles. When the shells interpenetrate, the overlapping region turns unstable, triggering the shock formation. As a first step towards a microscopic understanding of the process, we analyze here in details the initial instability phase. On the one hand, 2D relativistic PIC simulations are performed where two symmetric initially cold pair plasmas collide. On the other hand, the instabilities at work are analyzed, as well as the field at saturation and the seed field which gets amplified. For mildly relativistic motions and onward, Weibel modes with $\omega=0+i \delta$ govern the linear phase. We derive an expression for the duration of the linear phase in reasonable agreement with the simulations.
\end{abstract}

\section{Introduction}

The Fireball scenario for Gamma-Rays-Bursts (Nakar 2007) relies on shock particle acceleration (Blandford \& Ostriker 1978), where the shock arises from the encounter of two ultra-relativistic plasma blobs ejected from a central engine.

The formation of a shock following the collision of two plasma shells is now numerically well documented (Spitkovsky 2008), and subsequent particle acceleration has been observed in simulations (Nishikawa et al. 2005). In addition, shock generation through counter-streaming plasmas has already been observed in laboratory (Joseph et al. 2011).

\footnotetext{
1 ETSI Industriales, Universidad de Castilla-La Mancha, 13071 Ciudad Real, Spain

2 GoLP/Instituto de Plasmas e Fusão Nuclear, Laboratório Associado, Instituto Superior Técnico, Lisboa, Portugal

3 CEA, DAM, DIF, 91297 Arpajon, France

4 Harvard-Smithsonian Center for Astrophysics, 60 Garden Street, MS-51 Cambridge, MA 02138, USA
} 


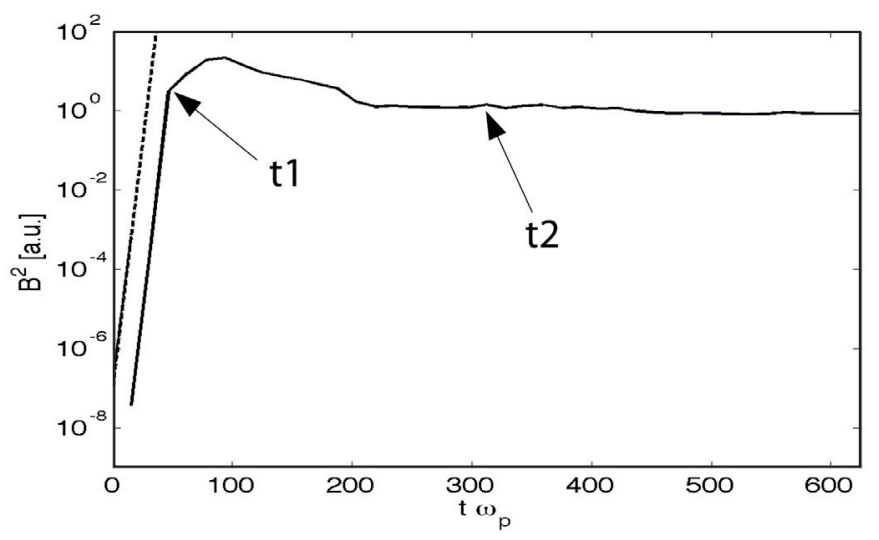

Fig. 1. Growth of the magnetic energy integrated over the transverse direction in the overlapping region. The dashed line is the theoretical growth-rate. The initial Lorentz factor was $\gamma_{0}=25$. All the field growth plots look qualitatively the same until $\gamma_{0}=10^{4}$. The saturation time $\tau_{s}$ is $t_{1}$. By this time, the density in the overlapping region is still twice the shells density. The density jump $\sim 3.3$ times then builds up, and the shock is formed around $t=t_{2}$. The field at saturation is $B\left(\tau_{s}\right)$.

Although the full shock formation process has been now repeatedly observed, a first principle understanding of the very birth of the shock is still lacking. Such an understanding could provide an accurate timing of the shock formation time, and constraints the conditions required to form it in the first place. Whether they are in the lab, in a computer or in the vicinity of a supernova, it should be possible to separate the scenario leading to the shock into two phases. In the first phase, plasma shells make contact, then overlap, and the overlapping region turns unstable. An instability grows and saturates. At this junction, the total density in the overlapping region is roughly the sum of each plasma density. A second phase is therefore needed during which nonlinear processes pick-up the system from the end of the linear phase, and build-up the Rankine-Hugoniot expected density jump near the borders of the interpenetrating shells.

The present paper is concerned with the first of these two phases. The collision of two identical cold relativistic pair plasmas has been simulated in $2 \mathrm{D}$ with the PIC Code OSIRIS. This setup has been chosen for its simplicity, allowing for a neat comparison with theory as the only free parameter is the initial Lorentz factor of the shells $\gamma_{0}$. Periodic boundary conditions are applied in the transverse direction. The evolution of the magnetic field in the overlapping region is displayed on Figure 1 for $\gamma_{0}=25$. The saturation time $\tau_{s}$ ( $t_{1}$ on the figure) is defined as the end of the exponential growth of the field energy integrated over the overlapping region. The field at saturation is simply $B\left(\tau_{s}\right)$.

As it amplifies a seed field from its initial fluctuation value to saturation, the instability governs this first phase of the shock formation for a time $\tau_{s}$ that we labeled "saturation time". A theoretical determination of this time which 
represents a lower bound to the shock formation time, is the main result of this paper.

\section{Instability analysis}

We here deal with the first phase of the shock formation, namely the instability of the overlapping region. Indeed, if counter streaming collisionless plasmas were not unstable, they would simply go through each other without anything happening.

The full unstable $\mathbf{k}$ spectrum has been analyzed long ago in the cold regime, where a shell is much denser than the other (Watson et al. 1960). These early results were recently generalized to the hot symmetric case (Bret \& Deutsch 2005; Bret et al. 2010). For wave-vectors aligned with the flow, we find two-stream unstable modes. Then, for wave-vectors normal to the flow, we find the filamentation, or Weibel, instability. Finally, modes propagating at arbitrary angle with the flow are also unstable (Bret et al. 2004). As the two plasmas penetrate each other, all the modes are excited. But the fastest growing one quickly overcomes the other, and shapes the linear phase. For the case we consider, a calculation of the growth-rate for every possible k's shows that only oblique and filamentation instabilities can dominate. The transition from oblique to filamentation occurs for $\gamma_{0}=\sqrt{3 / 2}$ (Bret et al. 2012). Beyond this threshold, filamentation governs the interaction with a growth rate,

$$
\frac{\delta}{\omega_{p}}=\frac{v_{0}}{c} \sqrt{\frac{2}{\gamma_{0}}} \sim \sqrt{\frac{2}{\gamma_{0}}} .
$$

Comparing this value with the growth of the field observed in the overlapping region results in a very satisfactory agreement, as evidenced in Figure 1. We also verified that the Weibel/Oblique transition does occur around $\gamma_{0}=\sqrt{3 / 2}$.

Noteworthily, it has been know for long that a flow aligned magnetic field can cancel the Weibel instability. But recent works performed in the cold regime proved the instability can never be canceled this way, providing the field is not perfectly aligned (Bret \& Pérez-Álvaro 2011).

\section{Saturation time}

Knowing the growth-rate (2.1) should allow for an accurate timing of the linear phase. Assuming that the instability amplifies a seed field of amplitude $B_{i}$ up to a saturation level $B_{s}$, we can write for the saturation time $\tau_{s}$,

$$
B_{f}=B_{i} e^{\delta \tau_{s}} \Rightarrow \tau_{s}=\frac{1}{2 \delta} \ln \left(\frac{B_{f}^{2}}{B_{i}^{2}}\right)
$$

where, for convenience, we consider the field energy $B^{2}$ ratio, instead of the field itself. Determining the saturation time amounts then to determine the initial and final fields. 
One way to derive the value of the saturation field $B_{f}=B\left(\tau_{s}\right)$, consists in stating that the field grows exponentially as long as it is small enough for the system to fit the linear approximation. Since a field $B_{f}$ affects particles on a time scale given by the cyclotron frequency, this implies Medvedev \& Loeb (1999),

$$
\frac{q B_{f}}{\gamma_{0} m c}=\delta \Rightarrow B_{f}=\frac{\gamma_{0} m}{q} \delta c .
$$

Accounting in addition for the growth-rate expression (2.1) gives,

$$
B_{f}^{2}=2 \gamma_{0}\left(\frac{m c \omega_{p}}{q}\right)^{2}
$$

which fits very well the values observed in the simulations.

Regarding the initial field amplitude, the idea is that the instability mechanism picks up a seed field from the spontaneous fluctuations of the system, and amplifies it. Various authors have been dealing with plasma fluctuations (Sitenko 1967), and the ability of PIC simulations to correctly render them has been checked by (Dieckmann et al. 2004) in the non-relativistic electrostatic case. Inserting the resulting $B_{i}$ in Equation (3.1) for the saturation time,

$$
\tau \omega_{p}=\frac{\sqrt{\gamma_{0}}}{2 \sqrt{2}} \ln \left[\frac{n\left(c / \omega_{p}\right)^{3}}{\sqrt{2} \pi} \frac{\mu \gamma_{0}^{3 / 2}}{\frac{15}{4 \gamma_{0}}+\mu \ln \left[\frac{1+4 \gamma_{0} / \mu}{1+\gamma_{0} / 4 \mu}\right]}\right], \quad \mu=\frac{m c^{2}}{k_{B} T},
$$

where $n$ is the plasma density.

Simulations have been run with nearly cold colliding plasma shells, with thermal velocity $\mu=10^{6}$. The time to reach saturation observed from the simulations is compared on Figure 2 with Equation (3.4). Clearly, the scaling is correct while a factor 1.7 is consistently laking.

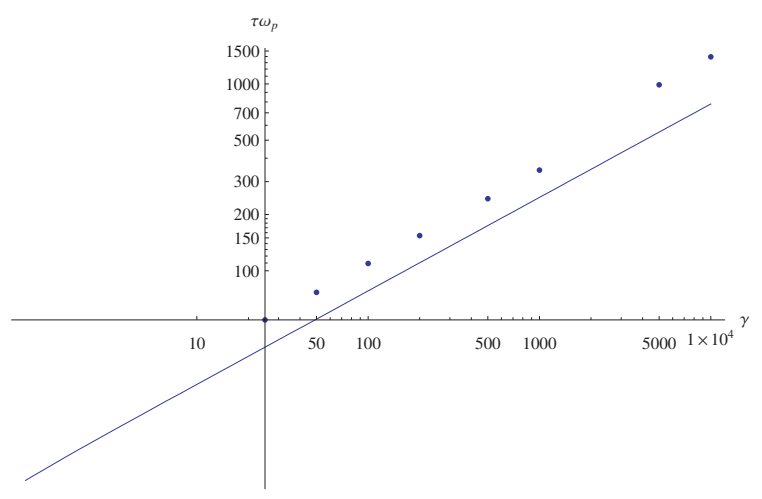

Fig. 2. Saturation time in $\omega_{p}$ units measured from simulations, circles, vs. Equation (3.4). 


\section{Conclusion}

This work represents a first step towards a first principles understanding of a collisionless shock formation. The equation derived already represents a lower bound to the shock formation time. Multiplied by $c$, it just tells how large the colliding plasma shells must be, if a shock is to be formed.

Future works focused on the second phase of the shock formation, namely the building-up of the density jump, should provide a theory of the full process hopefully able to constraint progenitors parameters in GRB's physics.

\section{References}

Nakar, E., 2007, Phys. Rep., 442, 166

Blandford, R., \& Ostriker, J., 1978, ApJ, 221, L29

Spitkovsky, A., 2008, ApJ, 682, L5

Nishikawa, K.-I., Hardee, P., Richardson, G., et al., 2005, ApJ, 622, 927

Joseph, J.A., Thomas, J.E., Kulkarni, M., et al., 2011, Phys. Rev. Lett., 106, 150401

Faŭnberg, Y.B., Shapiro, V.D., \& Shevchenko, V., 1970, Soviet Phys. JETP, 30, 528

Watson, K.M., Bludman, S.A., \& Rosenbluth, M.N., 1960, Phys. Fluids, 3, 741

Bret, A., \& Deutsch, C., 2005, Phys. Plasmas, 12, 082704

Bret, A., Gremillet L., \& Dieckmann, M.E., 2010, Phys. Plasmas, 17, 120501

Bret, A., Firpo M.-C., \& Deutsch, C., 2004, Phys. Rev. E, 70, 046401

Bret, A., Stockem A., Fiuza F., et al., 2012, Submitted to Phys. Plasmas

Bret, A., \& Pérez-Álvaro, E., 2011, Phys. Plasmas, 18, 080706

Medvedev, M.V., \& Loeb, A.A., 1999, ApJ, 526, 697

Sitenko, A.G., 1967, Electromagnetic Fluctuations in Plasma (Academic Press, New York)

Dieckmann, M.E., Ynnerman, A., Chapman, S.C., et al., 2004, Phys. Scripta, 69, 456 
